RLELNE TEXTE FƯR VORLESUNGEN UND UBUNGEN HERAUSGEGRBEN VON HANS LIETZMANN

\title{
SVPPLEMENTVM SOPHOCLEVM
}

EDIDIT

ERNESTVS DIEHL

BONN

A. MARCUS UND E. WRBER'S VERLAG

1913 
Supplemento Sophocleo noua fabularum poetae inlustris frustula et fragmenta e papyris et codicibus hausta congessimus eaque certa et uno uu. dd. consensu Sophocli uindicata: Indagatorum igitur et Eurypyli et Achaeorum conuentus papyros hoc libello edituri sumus et fragmenta quae ad lexici Photiani codicem Berolinensem publici iuris primus fecit Riccardus Reitzenstein et minora quaedam e scholiis uetustis et papyris nupernime in lucem prolata. omnino ea tragoediarum frustula negleximus, quae coniectura parum certa Sophocli tribuerunt uu. dd. uelut Chrysae quod agnouisse sibi uisus est fabulae fragmentum O. Rossbach, Berl. philol. Wochenschr. I899, I630ss, Niobes tragoediae cuiusdam uestigia Oxyrhynch. Pap. II nro. I23 (cf. G. E. Rizzo, Rivista di filologia classica 30, 1902, 462 ss), fragmenta illa tragica papyracea, quae a Grenfell et Hunt Pap. Hibeh I 1906, I7 ss primum edita Fr. Blass Tyrus Sophocleae esse censuit. 